\title{
Activating Standardization Bodies Around Medical Apps
}

\author{
Michael J. Ackerman ${ }^{1}$ \\ ${ }^{1}$ U.S. National Library of Medicine Bethesda
}

\begin{abstract}
The accuracy and safety of the information provided by medical and health related apps are of concern to medical and healthcare professionals. In the United States medical and certain health related devices are regulated for safety and accuracy by the U.S. Food and Drug Administration (FDA). Wireless devices, like smart phones, are regulated by the US Federal Communications Commission (FCC). Until now, the approval process was limited to complete devices. An FDA regulated medical device can contain software but the regulatory approval process involves the complete device, not each of its components separately. But what about apps? Apps are software which may turn an otherwise non-medical or non-healthcare device into a medical or healthcare device.
\end{abstract}

Do you regulate the software? It only has value when running on a device. Should you regulate the combination of device and software? It seems that every day a new smart phone is introduced and new apps become available. Do you have to test every combination? This paper will discuss the approach being taken in the United States by the government as well as by private industry.

\section{Keywords}

m-Health, Portable Software Apps, Medical Devices, Telemedicine, Mobil Phone

EJBI 2015; 11(3):en4-en6

received: June 13, 2014

accepted: October 10, 2014

published: April 20, 2015

\section{Introduction}

Traditionally one judged the content of a book or other reading material by its cover or place of purchase. Then the Web happened and reading material no longer had covers. In a similar sense one judged application software by the developer or the place of purchase. And then apps happened and there is no longer a known developer and sometimes not even a recognized place of purchase. How does one know that the app does what it claims to do? If it does not, who is responsible? One could argue that for less the $\$ 1.00$ US it really doesn't matter. Try it and see if it works. If it does - great! If it doesn't - so what, it only cost $\$ 1.00$ US to find out.

This approach works for most instances. But in the instance of medical apps such an approach could cause harm. If the medical app makes a recommendation based on some form of automatic or manual input and if that recommendation is inaccurate or just plain wrong, a dangerous situation could result for the user.

\section{The Problem}

Here is the scope of the problem: A medical app is developed in Malaysia, purchased from the iTunes store located in the United States by a person located in Italy for use in Brazil. Who is the source of trust for the information or advice given by this medical app, the equivalent of the old book's cover? And who is responsible if there is a problem?

For medical devices in the past we have turned to national and international regulatory and standardization agencies like the US Food and Drug Administration (FDA), the US Federal Communications Commission (FCC), the European Committee for Standardization (CEN) or the International Standards Organization (ISO). But these agencies tend to take their time whereas the apps business is almost an overnight business. The development time of an app and the work flow of these agencies is a complete mismatch.

The problem of the medical app has arisen from the new phenomenon of m-Health (mobile health). M-Health can be seen as the practice of medicine and public health, supported by mobile devices, such as smart phones and wireless connectivity, for health services and information. 
An alternative, perhaps a more popular definition, is the use of mobile devices to support health related monitoring and self-help activities.

In November 2012 the Pew Research Center, as part of its Internet Project, published a report entitled Mobil Health 2012 [1] that documented the prevalence of accessing health information through the use of a smart phone in the US. The report pointed out that $52 \%$ of smart phone owners gather health information using their phones. Latinos, African Americans, people between the ages of 18-49, or people who hold a college degree are more likely to gather health information through a smart phone. Women between the ages of 30 and 64 are more likely to have signed up for health text alerts. In 2012, $20 \%$ of smart phone owners already had at least one health app. Exercise, diet and weight related apps were the most popular.

\section{Who is Responsible?}

In the US wireless devices are regulated by the FCC, medical devices are regulated by the FDA. But who will regulate a wireless medical device?

In September 2012 the FCC m-Health Task Force made recommendations concerning who should be responsible for m-Health 2]. The general recommendation was that the FCC should continue to play a leadership role in advancing mobile health adoption. But the specific recommendations dealt with communication devices, communication links and access to communication links and devices. The question of m-Health software, medical apps, went unanswered.

\section{An Uneasy Solution}

One year later, in September 2013, the FDA published its advice Mobile Medical Applications: Guidance for Industry and Food and Drug Administrative Staff [3]. The FDA stated that it would regulate $m$-Health apps that provide diagnostic or clinical decision support, that convert a device into an accessory for an already regulated medical device or into a regulated medical device. Examples of accessory devices included remote display of ECG waveforms or medical images like pathology or radiology, or the control of a blood pressure cuff or an insulin pump. Examples of medical devices included sleep monitors, ECGs, tremor and balance monitors, and eye movement analyzers.

The FDA went on to describe a category of m-Health software that it does not expect to regulate but is reserving the right to do so - m-Health apps that manage health status. Included in this category are m-Health apps that: help users self-manage their wellness without providing suggestions or support to avoid disease such as managing weight or salt intake, or calculating the body-mass index (BMI); provide simple tools to organize and track health information such as data logs for allergies, obesity, heart disease or fitness; help document communications with a health provider; coach patients on how to cope with a disease such heart disease, high blood pressure, or diabetes but not how to treat it; provide access to health information such as medical dictionaries, textbooks, data bases, or web sites; and provide access and interaction with the patient's electronic health record (EHR) and personal health record (PHR).

If one were to summarize the FDA guidance one could conclude that the FDA will exercise its regulatory authority over a device or program which provides medical recommendations. Unfortunately this raises additional questions. When does the advice offered by an m-Health app fall into the regulated category of disease claims versus the unregulated category of wellness claims? When is an m-Health app an accessory to a medical device versus part of the communications network attached to that device? A tremendous number of m-Health apps are said to be designed to help us live healthier lives as opposed to managing a disease.

\section{The Reality}

The attempt to maintain the difference between disease and wellness can be seen on various software and device web sites. For example Owlet [4], a Bluetooth based sensor device that communicates with an m-Health smart phone app. The Owlet web site states that a Smart Sock (the device) is worn by a baby as he or she sleeps. The Smart Sock collects heart rate, oxygen, and sleep data and sends it via Bluetooth 4.0 to the parent's smart phone. The infant's data is then pushed to the cloud by the smart phone. The website clearly states This is not a medical device. This is for health and wellness purposes only. The statement may not be needed according the FDA advisory in that the sensor device is just collecting data and the mHealth app is just acting as a communicator between the sensor and the cloud. So the part of the system which needs oversight is the data interpretation system in the cloud and that system is not identified.

Newer m-Health apps and more advanced smart phones are making the distinctions made in the FDA guidance appear even less discernable. M-Health apps have been developed which use the smart phone's microphone to measure heart rate and the phone's accelerometers to measure tremor. Smart phones are now being developed that have built in EKG sensors. It would appear that the distinction boils down to a single criterion: Does the app make a medical recommendation?

As already noted, the majority of m-Health apps don't make medical recommendations, they loosely give wellness advice. In a paper published in the Journal of the American Medical Association, Powel, Landman and Bates [5] proposed a neutral third party as an internationally recognized testing and certification body following the Health on the Net (HON) Foundation 6] model which is often used by health related web sites. The problem may be 
the business plan. At less the $\$ 1.00$ US per download, will the developers support such an endeavor? If they will not, who will pay for the service?

The experiment was tried by a company named Happtique [7, a for-profit arm of the non-profit Greater New York Hospital Association. In late February 2013, Happtique published final standards for its m-Health Application Certification Program (HACP) designed to serve as a "good housekeeping seal of approval" for m-Health apps. HACP was designed to evaluate and certify m-Health apps for privacy, security and content. In June, Happtique announced a collaboration with the Association of American Medical Colleges (AAMC) and the Commission on Graduates of Foreign Nursing Schools to evaluate the m-Health apps.

Happtique's business plan included a $\$ 2,500$ US to $\$ 3,000$ US charge for each app evaluated. Apps would be evaluated within 30 days of submission. The certification program would determine if a given app: met stated data security standards; operated as intended; protected user privacy; and contained credible content. In November 2013 HACP certified its first cohort of m-Health apps.

It would have been nice if this was the end of the story and HACP became the solution to the m-Health app problem. But on December 13, 2013 Happtique had to suspend the program, less than two weeks after it approved the first cohort of m-Health apps. The problem, security issues were discovered in two of the apps it had just certified as secure. The result was a disappointing and embarrassing end to a program that was designed to boost physicians' confidence in m-Health apps to the point where they would feel comfortable prescribing them to patients.

\section{Perspective}

In the United States, m-Health and m-Health apps are likely to take a non-medical track. The FDA has clearly stated that they intend to regulate medical apps and will probably not look after health apps. Health insurance in the United States is through a private rather than government payer system. The system is resistant to pay for anything which is not considered required medical. Health products are paid for by the purchaser with no reimbursement. The entire food supplement and health food industry is based on this system and the advertising for and label of each of the products clearly states This product is not intended to diagnose, treat, cure, or prevent any disease. The public is willing to accept the implied risk and gladly pays for the products. The business model for non-medical m-Health apps will likely follow the already established non-medical health industry model.

\section{Acknowledgements}

This research was supported by the Intramural Research Program of the National Library of Medicine, NIH.

\section{References}

[1] Fox, Susannah and Duggan, Maeve. Mobile Health 2012, Pew Research Internet Project, November 2012. http://www . pewinternet.org/2012/11/08/mobile-health-2012/

[2] Goldman, J., J. Robert, D. Trauner. Mhealth Task Force: Findings and Recommendations, US Federal Communications Commission, September 2012. http://www.itif.org/events/ recommendations-mhealth-task-force

[3] Mobile Medical Applications: Guidance for Industry and Food and Drug Administrative Staff, US Food and Drug Administration, September 2013. http://www.fda.gov/ downloads/MedicalDevices/DeviceRegulationandGuidance/ GuidanceDocuments/UCM263366.pdf

[4] http://owletcare.com/

[5] Powel, AC, AB Landman, DW Bates. JAMA, doi:10.1001/jama, 3/24/2014.

[6] http://www.hon.ch/

[7] http://www.happtique.com/ 